\title{
QUADRATURE FORMULAE AND FUNCTIONS OF EXPONENTIAL TYPE
}

\author{
QAZI I. RAHMAN AND GERHARD SCHMEISSER
}

\begin{abstract}
In this paper we obtain certain generalizations of the trapezoidal rule and the Euler-Maclaurin formula that involve derivatives. In the case of quadrature of functions of exponential type over infinite intervals we find conditions under which existence of the (improper) integral and convergence of the approximating series become equivalent. In the process, we also establish a best possible version of a theorem of R. P. Boas and A. C. Schaeffer.
\end{abstract}

\section{INTRODUCTION AND SOME NOTATIONS}

The following result was recently proved by Olivier and Rahman [11]; also see [8].

Theorem A. Let $m$ be a nonnegative integer. Further, let $c_{0,0}=1$, whereas for $m>1$ and $0 \leq \mu \leq m$, let $c_{m, 2 \mu}$ be given by

$$
Q_{m}(x):=\prod_{j=1}^{m}\left(1+\left(\frac{x}{2 \pi j}\right)^{2}\right)=\sum_{\mu=0}^{m} c_{m, 2 \mu} x^{2 \mu} .
$$

If $f$ is an entire function of exponential type less than $2 \pi(m+1)$, then

$$
\int_{-\infty}^{+\infty} f(x) d x=\sum_{\mu=0}^{m} c_{m, 2 \mu} \sum_{n=-\infty}^{\infty} f^{(2 \mu)}(n),
$$

provided the integral on the left (taken in the sense of Cauchy) and the $m+1$ series on the right are convergent.

It may be recalled that

$$
\int_{a}^{+\infty} f(x) d x
$$

is said to exist in the sense of Cauchy if

$$
\lim _{x \rightarrow+\infty} \int_{a}^{X} f(\xi) d \xi
$$

exists; further, $\int_{-\infty}^{+\infty} f(x) d x$ exists in the sense of Cauchy if $\int_{0}^{+\infty} f(x) d x$ and $\int_{0}^{+\infty} f(-x) d x$ both exist in the sense of Cauchy.

Received April 21, 1988.

1980 Mathematics Subject Classification (1985 Revision). Primary 65D30, 65D32; Secondary 30D99. 
Since we are dealing with entire functions of exponential type, it is natural to wonder if the requirement about the convergence of the series was not really redundant. That it may indeed be superfluous is suggested by the following result of Boas and Schaeffer (applied to $\int_{a}^{z} f(\zeta) d \zeta$ ).

Theorem B [2]. Let $f$ be an entire function of exponential type $\pi$. Then $f(x)$ approaches a limit as $x \rightarrow+\infty$ if and only if

$$
\sum_{k=1}^{\infty} f^{\prime}(k+x)
$$

converges uniformly for $0 \leq x \leq 1$.

Although Theorem B does not solve our problem, even in the case $m=0$, it does offer some hope because it was not claimed to be the best possible result of its kind. This has served as a motivation for us to obtain the sharp version of Theorem B (see Corollary 2) and look for generalizations (hopefully) adequate to handle the question concerning the convergence of the $m+1$ series in (1.2) for arbitrary $m$.

Here are some other questions which may be asked with reference to Theorem A:

Q1. Is there an analogue of Theorem $\mathrm{A}$ for integrals over $(0,+\infty)$ ? The function may be assumed to be of (appropriate) exponential type in the right half-plane.

Q2. Are there any analogues of Theorem A for integrals over finite intervals? If so, how are they related to some of the well-known quadrature formulae like the Euler-Maclaurin formula?

We follow an alternative approach to Theorem A which turns out to be appropriate for the problem of Theorem B as well. It is based on the time-honored calculus of residues [9] - a technique which has already been used to obtain representations for the remainders of quadrature formulae and to consider various questions which arise in their study $[10,15,4,13,14]$. This approach also allows us to treat the two questions just mentioned.

Before stating our results, it seems desirable to mention some of the notations we use. We write

$$
f_{m}(z):=\sum_{\mu=0}^{m} c_{m, 2 \mu} f^{(2 \mu)}(z),
$$

where the numbers $c_{m, 2 \mu}$ are defined by (1.1). In order to denote the error (in a quadrature formula) we use

$$
\begin{gathered}
E_{m}\left[M-\frac{1}{2}, N-\frac{1}{2} ; f\right]:=\int_{M-1 / 2}^{N-1 / 2} f(x) d x-\sum_{n=11}^{N-1} f_{m}(n), \\
E_{m}[M, N ; f]:=\int_{M}^{N} f(x) d x-\frac{1}{2} f_{m}(M)-\sum_{n=11+1}^{N-1} f_{m}(n)-\frac{1}{2} f_{m}(N),
\end{gathered}
$$


where $M$ and $N$ are integers. We have chosen to set

$$
\varphi(z):=1 /\left(1-e^{2 \pi i z}\right)
$$

and

$$
P_{m}(x)=\frac{(2 m+1) !}{(m !)^{2}} \int_{0}^{x} u^{m}(1-u)^{m} d u=\sum_{\mu=m+1}^{2 m+1} a_{\mu} x^{\mu}
$$

By $B_{2 k}$ we denote the $2 k$ th Bernoulli number and by $\widehat{B}_{2 k}(\cdot)$ the Bernoulli function obtained by restricting the Bernoulli polynomial of degree $2 k$ to the interval $[0,1]$ and continuing it with period 1 on the whole real line. Further, we set

$$
A_{1,2 j}:=\sum_{\mu=0}^{j^{*}} \frac{B_{2 j-2 \mu}}{(2 j-2 \mu) !}\left(1-2^{-2 j+2 \mu+1}\right) c_{m, 2 \mu}
$$

and

$$
A_{2,2 j}:=\sum_{\mu=0}^{j^{*}} \frac{B_{2 j-2 \mu}}{(2 j-2 \mu) !} c_{m, 2 \mu}
$$

where $j^{*}=\min \{m, j\}$.

\section{Statement of Results}

2.1. Two basic formulae. Our investigations are based on two formulae contained in Theorems 1 and $\mathrm{C}$.

Theorem 1. Let $T>0$ be a real number and let $M, N$ be integers such that $M<N$. If $f$ is holomorphic in the rectangle

$$
\mathscr{R}_{1}:=\left\{z \in \mathbf{C}: M-\frac{1}{2} \leq \operatorname{Re} z \leq N-\frac{1}{2},|\operatorname{Im} z| \leq T\right\},
$$

then in the notations (1.7) and (1.8) we have

$$
\begin{array}{rl}
\int_{M-1 / 2}^{N-1 / 2} & f(x) d x-\sum_{n=M}^{N-1} f_{m}(n) \\
= & i \int_{0}^{T} P_{m}\left(\frac{1}{1+e^{2 \pi t}}\right)\left\{f\left(N-\frac{1}{2}+i t\right)-f\left(N-\frac{1}{2}-i t\right)\right\} d t \\
& -i \int_{0}^{T} P_{m}\left(\frac{1}{1+e^{2 \pi t}}\right)\left\{f\left(M-\frac{1}{2}+i t\right)-f\left(M-\frac{1}{2}-i t\right)\right\} d t \\
& -\int_{M-1 / 2}^{N-1 / 2} P_{m}(\varphi(u-i T)) f(u-i T) d u \\
& -\int_{M-1 / 2}^{N-1 / 2} P_{m}(\varphi(-u-i T)) f(u+i T) d u .
\end{array}
$$

Theorem 1 contains an cpen quadrature formula. The corresponding closed formula exists for $m=0$ only. It is the trapezoidal rule for functions holomorphic in a rectangle. We state it as Theorem $\mathrm{C}$ and refer the reader to [6, $\S 13.14]$ and [10] for proof and comments. 
Theorem C. Let $T>0$ be a real number and let $M, N$ be integers such that $M<N$. If $f$ is holomorphic in the rectangle

$$
\mathscr{R}_{2}:=\{z \in \mathbf{C}: M \leq \operatorname{Re} z \leq N,|\operatorname{Im} z| \leq T\},
$$

then

$$
\begin{aligned}
\int_{M}^{N} f(x) d x-\frac{1}{2} f(M)-\sum_{n=M+1}^{N-1} f(n)-\frac{1}{2} f(N) \\
=i \int_{0}^{T} P_{0}\left(\frac{1}{1-e^{2 \pi t}}\right)\{f(N+i t)-f(N-i t)\} d t \\
\quad-i \int_{0}^{T} P_{0}\left(\frac{1}{1-e^{2 \pi t}}\right)\{f(M+i t)-f(M-i t)\} d t \\
\quad-\int_{M}^{N}\left\{P_{0}(\varphi(u-i T)) f(u-i T)+P_{0}(\varphi(-u-i T)) f(u+i T)\right\} d u
\end{aligned}
$$

2.2. Quadrature over finite intervals. The formulae (2.1) and (2.2) in their present form are not convenient for numerical quadrature since their remainders $E_{m}\left[M-\frac{1}{2}, N-\frac{1}{2} ; f\right]$ and $E_{m}[M, N ; f]$ may not be small. We therefore need to modify the approximants so that they may allow the integral to be calculated with any desired degree of precision. With this objective in mind, we will study the two formulae further in the preeminently important case $T \rightarrow 0$. The case $T \rightarrow \infty$ is also of interest to us and will be discussed in subsection 2.4.

Theorem 2. Let $N$ and $k$ be integers such that $N \geq 1$ and $k \geq m$. If $f:[a, b] \rightarrow \mathbf{C}$ is $2 k$ times continuously differentiable, then in the notations of (1.1) and (1.9) we have

$$
\begin{array}{rl}
\int_{a}^{b} & f(x) d x-\sum_{\mu=0}^{m} h^{2 \mu+1} c_{m, 2 \mu} \sum_{n=0}^{N-1} f^{(2 \mu)}\left(a+\frac{2 n+1}{2} h\right) \\
& -\sum_{j=1}^{k} h^{2 j} A_{1,2 j}\left\{f^{(2 j-1)}(b)-f^{(2 j-1)}(a)\right\}=R_{1, k, m, N}[f],
\end{array}
$$

where $h=(b-a) / N$ and (expressed in terms of the Bernoulli functions)

$$
R_{1, k, m, N}[f]=h^{2 k} \int_{a}^{b} f^{(2 k)}(x) \sum_{\mu=0}^{m} c_{m, 2 \mu} \frac{\widehat{B}_{2 k-2 \mu}\left(\frac{x-a}{h}-\frac{1}{2}\right)}{(2 k-2 \mu) !} d x .
$$

Theorem 3. Let $N$ and $k$ be integers such that $N \geq 1$ and $k \geq m$. If $f:[a, b] \rightarrow \mathbf{C}$ is $2 k$ times continuously differentiable, then in the notations of (1.1) and (1.10) we have

$$
\begin{aligned}
\int_{a}^{b} f(x) d x & -\sum_{\mu=0}^{m} h^{2 \mu+1} c_{m, 2 \mu}\left\{\frac{1}{2} f^{(2 \mu)}(a)+\frac{1}{2} f^{(2 \mu)}(b)+\sum_{n=1}^{N-1} f^{(2 \mu)}(a+n h)\right\} \\
& +\sum_{j=1}^{k} h^{2 j} A_{2,2 j}\left\{f^{(2 j-1)}(b)-f^{(2 j-1)}(a)\right\}=R_{2, k, m, N}[f],
\end{aligned}
$$


where $h=(b-a) / N$ and (expressed in terms of the Bernoulli functions)

$$
R_{2, k, m, N}[f]=h^{2 k} \int_{a}^{b} f^{(2 k)}(x) \sum_{\mu=0}^{m} c_{m, 2 \mu} \frac{\widehat{B}_{2 k-2 \mu}\left(\frac{x-a}{h}\right)}{(2 k-2 \mu) !} d x .
$$

Remark 1. Formula (2.4) may be thought of as a generalization of the EulerMaclaurin formula since it reduces to the latter if $m=0$.

The remainders $R_{j, k, m, N}[f](j=1,2)$ in Theorems 2 and 3 can be controlled by the choice of $k, m$ and $N$. For $R_{2, k, m, N}[f]$ we present an alternative representation which shows the influence of $k, m$ and $N$ explicitly.

Corollary 1. Let the function $f$ in Theorem 3 be $2 k+2$ times continuously differentiable. Defining

$$
M_{k, m}(x):=\sum_{\mu=0}^{m} \frac{\widehat{B}_{2 k-2 \mu}(x)}{(2 k-2 \mu) !} c_{m, 2 \mu},
$$

we have

$$
R_{2, k, m, N}[f]=(a-b)\left(\frac{b-a}{N}\right)^{2 k+2} M_{k+1, m}(0) f^{(2 k+2)}(\xi),
$$

where $\xi \in[a, b]$. Moreover,

$$
\left|R_{2, k, m, N}[f]\right|
$$

$$
\leq \frac{2(b-a)}{(m !)^{2}}\left(\frac{b-a}{2 \pi N}\right)^{2 k+2} \frac{2 k+2-m}{2 k+1-2 m} \frac{1}{(m+1)^{2 k+2-2 m}} \max _{a \leq x \leq b}\left|f^{(2 k+2)}(x)\right| \text {. }
$$

2.3. Problems of convergence. Later on we shall study Theorems 2 and 3 for $b$ and $N$ tending to $\infty$ while $h=(b-a) / N$ remains fixed. Thus we shall arrive at an integral over an infinite interval and an infinite series of samples $f_{m}(n)$. Of course, we have to be sure that the integral exists and the series converges. This is what we investigate next. With the help of the first basic formula we are able to show that for functions of (appropriate) exponential type the existence of the integral and the convergence of the series imply each other under a (weak) side condition. As the first result in this direction we prove

Theorem 4. Let $f$ be holomorphic and of exponential type less than $2 \pi(m+1)$ in the right half-plane. If

$$
\lim _{x \rightarrow+\infty} \int_{1 / 2}^{x} f(t) d t
$$

exists, then in the notation of (1.4)

$$
\sum_{n=1}^{\infty} f_{m}(n+\lambda)
$$

converges uniformly for $\lambda \in[0,1]$.

Remark 2. We wish to point out that the uniform convergence of (2.9) does not imply the existence of the limit (2.8) if $m>0$. In fact, $f(z):=\cos (2 \pi m z)$ 
satisfies the differential equation

$$
\sum_{\mu=0}^{m} c_{m, 2 \mu} y^{(2 \mu)}=0
$$

as is seen from (1.1). Hence $f_{m}(z) \equiv 0$ so that (2.9) converges uniformly, whereas (2.8) does not exist. This is unlike Theorem B.

As a result in the opposite direction we have

Theorem 5. Let $f$ be holomorphic and of exponential type less than $2 \pi(m+1)$ in the right half-plane such that $f(x) \rightarrow 0$ as $x \rightarrow+\infty$. If

$$
\sum_{n=1}^{\infty} f_{m}(n)
$$

converges, then the limit (2.8) exists.

We now state a few consequences of Theorems 4 and 5, starting with a best possible improvement of Theorem B.

Corollary 2. Let $f$ be holomorphic and of exponential type less than $2 \pi$ in the right half-plane. Then $f(x)$ approaches a limit as $x \rightarrow+\infty$ if and only if (1.3) converges uniformly for $0 \leq x \leq 1$.

Remark 3. The function

$$
f(z)=\int_{0}^{z} \frac{\cos (2 \pi \zeta)}{\zeta-\frac{1}{4}} d \zeta
$$

is an entire function of exponential type $2 \pi$ and $\lim _{x \rightarrow+\infty} f(x)$ exists. However,

$$
\sum_{k=1}^{\infty} f^{\prime}(k)=\sum_{k=1}^{\infty} \frac{1}{k-\frac{1}{4}}
$$

does not converge. Hence the restriction on the exponential type in Corollary 2 cannot be relaxed.

For arbitrary $m \in \mathbf{N}_{0}$ we have to be content with the following simple consequence of Theorems 4 and 5 .

Corollary 3. Let $f$ be holomorphic and of exponential type less than $2 \pi(m+1)$ in the right half-plane. Then (2.8) exists if and only if (2.10) converges and $f(x) \rightarrow 0$ as $x \rightarrow+\infty$.

We next come to the main result of the paper, but first let us recall that

$$
\int_{-\infty}^{+\infty} f(x) d x
$$

exists as a Cauchy principal value, if

$$
\int_{0}^{+\infty}\{f(x)+f(-x)\} d x
$$


exists in the sense of Cauchy and

$$
\sum_{n=-\infty}^{+\infty} a_{n}
$$

exists as a Cauchy principal value if

$$
\sum_{n=1}^{\infty}\left(a_{n}+a_{-n}\right)
$$

converges.

Corollary 4. Let $f$ be an entire function of exponential type less than $2 \pi(m+1)$. Then

$$
\int_{-\infty}^{+\infty} f(x) d x
$$

exists as a Cauchy principal value if and only if $f(x)+f(-x) \rightarrow 0$ as $x \rightarrow+\infty$ and

$$
\sum_{n=-\infty}^{+\infty} f_{m}(n)
$$

exists as a Cauchy principal value. Moreover,

$$
\int_{-\infty}^{+\infty} f(x) d x=\sum_{n=-\infty}^{+\infty} f_{m}(n)
$$

in the sense of Cauchy principal values if (2.11) exists.

Remark 4. The quadrature formula (2.13) for entire functions of exponential type less than $2 \pi(m+1)$ and belonging to $L^{1}(\mathbf{R})$ could be deduced from a result of Kress [8]. Olivier and Rahman (Theorem A) proved it under the assumption that (2.11) exists in the sense of Cauchy and (2.12) converges. The condition imposed here is still weaker.

2.4. Quadrature of functions of exponential type in a vertical strip. As a first step towards obtaining quadrature formulae for functions of exponential type in a half-plane we find alternative representations for the remainders in Theorems 2 and 3 under the assumption that the function is holomorphic and of exponential type in a (vertical) strip.

Let

$$
S[a, b]:=\{z \in \mathbf{C}: a \leq \operatorname{Re} z \leq b\} .
$$

A function $f$ holomorphic in the strip $S[a, b]$ is said to be of exponential type less than $\tau>0$ if there exists an $\varepsilon>0$ such that

$$
|f(z)| \leq c e^{(\tau-\varepsilon)|z|}, \quad z \in S[a, b],
$$

with a constant $c>0$. 
Theorem 6. Let $N$ and $k$ be integers such that $N \geq 1$ and $k \geq m$. If $f$ is holomorphic and of exponential type less than $2(m+1) N \pi /(b-a)$ in $S[a-\delta, b+\delta]$ for some $\delta>0$, then in the notations of Theorem 2

$$
\begin{aligned}
R_{1, k, m, N}[f]=h^{2 k} \int_{0}^{+\infty} L_{1, k, m}\left(\frac{t}{h}\right) & \left\{f^{(2 k)}(b+i t)-f^{(2 k)}(b-i t)\right. \\
& \left.-f^{(2 k)}(a+i t)+f^{(2 k)}(a-i t)\right\} d t
\end{aligned}
$$

where

$$
L_{1, k, m}(t):=i(-1)^{k} \sum_{\nu=m+1}^{\infty}(-1)^{\nu} \frac{e^{-2 \pi t \nu}}{(2 \pi \nu)^{2 k}} Q_{m}(i 2 \pi \nu) .
$$

Theorem 7. Under the conditions of Theorem 6 and in the notations of Theorem 3 we have

$$
\begin{aligned}
R_{2, k, m, N}[f]=h^{2 k} \int_{0}^{+\infty} L_{2, k, m}\left(\frac{t}{h}\right) & \left\{f^{(2 k)}(b+i t)-f^{(2 k)}(b-i t)\right. \\
& \left.-f^{(2 k)}(a+i t)+f^{(2 k)}(a-i t)\right\} d t,
\end{aligned}
$$

where

$$
L_{2, k, m}(t):=i(-1)^{k} \sum_{\nu=m+1}^{\infty} \frac{e^{-2 \pi t \nu}}{(2 \pi \nu)^{2 k}} Q_{m}(i 2 \pi \nu) .
$$

2.5. Quadrature of functions of exponential type in a half-plane. Setting $a=0$, $b=N h$ and letting $N \rightarrow \infty$, Corollary 3 allows us to deduce from Theorems 6 and 7, respectively, the following two corollaries.

Corollary 5. Let $k \geq m$ be an integer and $h>0$ be a real number. Further, let $f$ be holomorphic and of exponential type less than $2 \pi(m+1) / h$ in the halfplane $\{z \in \mathrm{C}: \operatorname{Re} z \geq-\delta\}$, where $\delta$ is some positive number. If the integral of $f$ over $[0,+\infty)$ exists in the sense of Cauchy and the numbers $A_{1,2 j}$ are as in (1.9), then

$$
\begin{aligned}
\int_{0}^{+\infty} f(x) d x= & \sum_{n=0}^{\infty} \sum_{\mu=0}^{m} h^{2 \mu+1} c_{m, 2 \mu} f^{(2 \mu)}\left(\frac{2 n+1}{2} h\right) \\
& -\sum_{j=1}^{k} h^{2 j} A_{1,2 j} f^{(2 j-1)}(0)+\rho_{1, k, m, h}[f],
\end{aligned}
$$

where $\rho_{1, k, m, h}[f]$ is given in terms of the function $L_{1, k, m}$ of (2.17) by

$$
\rho_{1, k, m, h}[f]=-h^{2 k} \int_{0}^{+\infty} L_{1, k, m}\left(\frac{t}{h}\right)\left\{f^{(2 k)}(i t)-f^{(2 k)}(-i t)\right\} d t .
$$

Corollary 6. Under the assumptions of Corollary 5, we have

$$
\begin{aligned}
\int_{0}^{+\infty} f(x) d x= & \sum_{n=1}^{\infty} \sum_{\mu=0}^{m} h^{2 \mu+1} c_{m, 2 \mu} f^{(2 \mu)}(n h) \\
& +\sum_{j=0}^{2 k-1} h^{j+1} C_{j} f^{(j)}(0)+\rho_{2, k, m, h}[f],
\end{aligned}
$$


where in the notation of (1.10)

$$
C_{j}:= \begin{cases}\frac{1}{2} c_{m, j} & \text { for } j=0,2, \ldots, 2 m, \\ A_{2, j+1} & \text { for } j=1,3, \ldots, 2 k-1, \\ 0 & \text { in all other cases },\end{cases}
$$

and $\rho_{2, k, m, h}[f]$ is given in terms of the function $L_{2, k, m}$ of $(2.19)$ by

$$
\rho_{2, k, m, h}[f]=-h^{2 k} \int_{0}^{+\infty} L_{2, k, m}\left(\frac{t}{h}\right)\left\{f^{(2 k)}(i t)-f^{(2 k)}(-i t)\right\} d t .
$$

For $h=1$ and $k=m=0$ the formula of Corollary 6 reduces to the "summation formula of Plana" [7, p. 274]. For another quadrature formula over $[0,+\infty)$, see [13].

Example. Let $f$ be an entire function of exponential type $\tau$, real on the real line and bounded there by a constant $\mathscr{M}$, say. Then by Bernstein's inequality $\left|f^{(2 k+1)}(x)\right| \leq \mathscr{M} \tau^{2 k+1}$ for $x \in \mathbf{R}$ and by a result of Duffin and Schaeffer [1, Theorem $6.2 .6^{*}$ ],

$$
\left|f^{(2 k+1)}(x+i y)\right| \leq \mathscr{M} \tau^{2 k+1} \cosh \tau y \quad \text { for } x+i y \in \mathbf{C} .
$$

Now by integration we get

$$
\left|f^{(2 k)}(i t)-f^{(2 k)}(-i t)\right| \leq 2 \mathscr{M} \tau^{2 k}|\sinh \tau t| \leq 2 \mathscr{M} \tau^{2 k+1}|t| \cosh \tau t .
$$

Using this bound for estimating (2.24), we obtain after integration by parts

$$
\left|\rho_{2, k, m, h}[f]\right| \leq 2 \mathscr{M} h \cdot(\tau h)^{2 k+1} \sum_{\nu=m+1}^{\infty} \frac{4 \pi^{2} \nu^{2}+\tau^{2} h^{2}}{\left(4 \pi^{2} \nu^{2}-\tau^{2} h^{2}\right)^{2}} \frac{\left|Q_{m}(i 2 \pi \nu)\right|}{(2 \pi \nu)^{2 k}},
$$

where $h$ has to be chosen less than $2 \pi(m+1) / \tau$. Since

$$
\left|Q_{m}(i 2 \pi \nu)\right|=\nu^{2 m} \prod_{j=1}^{m} \frac{\nu^{2}-j^{2}}{j \nu^{2}} \leq \frac{\nu^{2 m}}{(m !)^{2}},
$$

the infinite series may be estimated by

$$
\begin{aligned}
& \frac{1}{(m !)^{2}} \frac{1}{(2 \pi)^{2 k+2}} \sum_{\nu=m+1}^{\infty} \frac{1+\tau^{2} h^{2} / 4 \pi^{2} \nu^{2}}{\left(1-\tau^{2} h^{2} / 4 \pi^{2} \nu^{2}\right)^{2}} \frac{1}{\nu^{2 k-2 m+2}} \\
& \leq \frac{1}{(m !)^{2}} \frac{1}{(2 \pi)^{2 k+2}} \frac{1+\tau^{2} h^{2} / 4 \pi^{2}(m+1)^{2}}{\left(1-\tau^{2} h^{2} / 4 \pi^{2}(m+1)^{2}\right)^{2}} \\
& \quad \times\left\{\frac{1}{(m+1)^{2 k-2 m+2}}+\int_{m+1}^{\infty} \frac{1}{x^{2 k-2 m+2}} d x\right\} \\
& =\frac{1}{(m !)^{2}} \frac{1}{(2 \pi)^{2 k+2}}\left(1-\frac{\tau h}{2 \pi(m+1)}\right)^{-2} \frac{2 k+2-m}{2 k+1-2 m} \frac{1}{(m+1)^{2 k-2 m+2}},
\end{aligned}
$$

which shows various possibilities to control the remainder. The upper bound for $\left|\rho_{2, k, m, h}[f]\right|$ obtained this way is also an upper bound for $\left|\rho_{1, k, m, h}[f]\right|$. 
If $f$ is given to be holomorphic in $H_{\delta}:=\{z \in \mathbf{C}: \operatorname{Re} z \geq-\delta\}$ for some $\delta>0$ and satisfies

$$
|f(x+i y)| \leq c e^{\tau|y|}, \quad x+i y \in H_{\delta},
$$

then, using (3.18) for the $(2 k+1)$ st derivative and taking $\gamma$ to be the circle of radius $\delta$ centered at $x+i y$, we find

$$
\left|f^{(2 k+1)}(x+i y)\right| \leq c e^{\tau \delta} \frac{(2 k+1) !}{\delta^{2 k+1}} e^{\tau|y|} \quad \text { for } x \geq 0 .
$$

Consequently,

$$
\begin{aligned}
\left|f^{(2 k)}(i t)-f^{(2 k)}(-i t)\right| & \leq 2 c e^{\tau \delta} \frac{(2 k+1) !}{\delta^{2 k+1}}\left(e^{\tau|t|}-1\right) \\
& \leq 4 c e^{\tau \delta} \frac{(2 k+1) !}{\delta^{2 k+1}} \tau|t| \cosh \tau t,
\end{aligned}
$$

which could be used in the same way as (2.25) to obtain an estimate for the remainder.

Remark 5. While we have assumed $f$ to be holomorphic in a strip or a halfplane or in the whole plane, functions having a finite number of isolated singularities may also be considered. The singularities will contribute to the residues, and the quadrature formula will have to be accordingly modified. Functions holomorphic in sectors containing the interval of integration can also be treated using appropriate contours and properties of the Phragmén-Lindelöf indicator function [1, Chapter 5].

2.6. Further results on the question of convergence. The following result is obtained by applying Corollary 4 to the function $f(z) \cdot \overline{f(\bar{z})}$.

Corollary 7. Let $f$ be an entire function of exponential type less than $\pi(m+1)$. Then $f \in L^{2}(\mathbf{R})$ if and only if $f(x) \rightarrow 0$ as $x \rightarrow \pm \infty$ and

$$
\sum_{n=-\infty}^{\infty} \sum_{\mu=0}^{m} c_{m, 2 \mu}\left(\frac{d^{2 \mu}}{d z^{2 \mu}} f(z) \cdot \overline{f(\bar{z})}\right)_{z=n}
$$

exists as a Cauchy principal value. Moreover, if $f \in L^{2}(\mathbf{R})$, then $\|f\|_{L^{2}(\mathbf{R})}^{2}$ is equal to the quantity (2.27).

In the case $m=0$, Corollary 7 reduces to a result in [14, Theorem 2.1, equation (2.7)].

Here is another consequence of Corollary 4.

Corollary 8. Let $f$ be an entire function of exponential type less than $2 \pi$ such that $\int_{-\infty}^{+\infty} f(t) d t$ exists in the sense of Cauchy. Then

$$
\sum_{n=-\infty}^{\infty} f^{(\mu)}(n)=0
$$

for all $\mu \geq 1$. 
By obvious modifications of the proofs of Lemma 4 and Theorems 4 and 5, we obtain

Theorem 8. Let $f$ be holomorphic and of exponential type less than $2 \pi(m+1)$ in the right half-plane. If

$$
\int_{1 / 2}^{x} f(t) d t
$$

remains bounded as $x \rightarrow+\infty$, then (2.9) has uniformly bounded partial sums for $\lambda \in[0,1]$.

Theorem 9. Let $f$ be holomorphic and of exponential type less than $2 \pi(m+1)$ in the right half-plane. If (2.10) has bounded partial sums and $f$ is bounded on $(0,+\infty)$, then $(2.29)$ is also bounded on this interval.

Theorems 8 and 9 may be compared with Theorem 3 of [2].

As we will briefly indicate, the proofs of Lemma 4 and Theorems 4 and 5 can be modified to obtain the following two results which are in the spirit of $[2$, Theorem 4].

Theorem 10. Let $f$ be holomorphic and of exponential type less than $2 \pi(m+1)$ in the right half-plane. If

$$
\lim _{x \rightarrow+\infty} \frac{1}{x} \int_{1 / 2}^{x} f(t) d t
$$

exists, then

$$
\lim _{N \rightarrow \infty} \frac{1}{N} \sum_{n=1}^{N} f_{m}(n+\lambda)
$$

exists uniformly for $\lambda \in[0,1]$ and is the same for each $\lambda$. Further, the limits in (2.30) and (2.31) are equal.

Theorem 11. Let $f$ be holomorphic and of exponential type less than $2 \pi(m+1)$ in the right half-plane. If

$$
\lim _{N \rightarrow \infty} \frac{1}{N} \sum_{n=1}^{N} f_{m}(n)
$$

exists and $f(x)=o(x)$ as $x \rightarrow+\infty$, then (2.30) exists, and the two limits in question are equal.

2.7. Applications to uniqueness. It seems interesting to us that the quadrature formula in Corollary 4 can be used to obtain certain uniqueness theorems for entire functions of exponential type. To place our results in perspective we recall

Theorem D. If $f$ is an entire function of exponential type less than $\pi(k+1)$ and $f(n)=f^{\prime}(n)=\cdots=f^{(k)}(n)=0$ for all integers $n$, then $f(z) \equiv 0$.

Theorem E. If $f$ is an entire function of exponential type less than $\pi(k+1)$ and $f^{(2 \mu)}(n)=0$ for $\mu=0,1, \ldots, k$ and all integers $n$, then

$$
f(z)=\sum_{\mu=0}^{k} \alpha_{\mu} \sin \pi \mu z
$$

where $\alpha_{0}, \alpha_{1}, \ldots, \alpha_{k} \in \mathbf{C}$. 
Theorem D is a trivial generalization of a classical result of Carlson [16, $\S 5.81]$ and Theorem $E$ is due to Brück [3]. In order to state our results, which are variants of the above two theorems, we need to introduce a

Notation. We use $S$ or $S_{1}, S_{2}, \ldots$ to denote sectors of the form

$$
\left\{z \in \mathbf{C}: \varphi_{1} \leq \arg z \leq \varphi_{2}\right\}
$$

with $0 \leq \varphi_{2}-\varphi_{1}<\pi$. In particular, $S$ may be the interval $[0,+\infty)$.

Theorem 12. Let $k$ be an odd integer and let $f$ be an entire function of exponential type less than $\pi(k+1)$ which does not grow faster than a polynomial on the real line. If $f^{(j)}(n)=0$ for $j=0, \ldots, k-1$ and $f^{(k)}(n) \in S$ for all integers $n$, then $f(z) \equiv 0$.

As is shown by the example $f(z)=\sin ^{k} \pi z$, Theorem 12 does not hold for even $k$.

Theorem 13. Let $f$ be an entire function of exponential type less than $\pi(k+1)$, where $k \in \mathbf{N}$, such that $f^{\prime}(x)-f^{\prime}(-x)$ tends to zero as $x \rightarrow+\infty$. Set

$$
j:= \begin{cases}k & \text { for odd } k \\ k+1 & \text { for even } k\end{cases}
$$

If

and

$$
f^{\left(2 \prime^{\prime}\right)}(n)=0 \quad(\nu=0, \ldots,(j-1) / 2)
$$

for all integers $n$, then

$$
f^{(2 \mu)}(n) \in S_{\mu} \quad(\mu=(j+1) / 2, \ldots, k)
$$

$$
f(z)=\sum_{\mu=0}^{k} \alpha_{\mu} \sin \pi \mu z,
$$

where $\alpha_{0}, \alpha_{1}, \ldots, \alpha_{k} \in \mathbf{C}$.

\section{LEMMAS}

In order to make the exposition as lucid as we can, we present many of the details in the form of lemmas. But first we shall bring in some further notations. The function

$$
K_{m}(z):=-i \frac{(2 m+1) !}{2^{2 m+1}(m !)^{2}} \sum_{\mu=0}^{m}\left(\begin{array}{c}
m \\
\mu
\end{array}\right) \frac{1}{2 \mu+1}(\cot \pi z)^{2 \mu+1}
$$

plays an important role in our work. We find it convenient to introduce for $k=0,1, \ldots$ the functions

$$
\Phi_{k}(z):=i^{k+1} \sum_{\nu=1}^{\infty}(-1)^{\nu} \frac{e^{-2 \pi i \nu z}}{(2 \pi \nu)^{k}},
$$




$$
\Psi_{k}(z):=i^{k+1} \sum_{\nu=1}^{\infty} \frac{e^{-2 \pi i \nu z}}{(2 \pi \nu)^{k}}
$$

defined wherever the series converge. Obviously,

$$
\Phi_{k}^{\prime}(z)=\Phi_{k-1}(z), \quad \Psi_{k}^{\prime}(z)=\Psi_{k-1}(z)
$$

wherever they are differentiable. Furthermore, it is known that [12, pp. 226227]

$$
\begin{gathered}
2 \operatorname{Im} \Psi_{2 k}(x)=-\frac{\widehat{B}_{2 k}(x)}{(2 k) !} \text { for } x \in \mathbf{R}, \\
\Psi_{2 k}(0)=-\frac{i}{2} \frac{B_{2 k}}{(2 k) !}
\end{gathered}
$$

and

$$
\Phi_{2 k}(0)=\Psi_{2 k}\left(\frac{1}{2}\right)=\frac{i}{2} \frac{B_{2 k}}{(2 k) !}\left(1-2^{-2 k+1}\right),
$$

where $\widehat{B}_{2 k}(\cdot)$ and $B_{2 k}$ have already been defined in $\S 1$.

Lemma 1. The meromorphic function $K_{m}$ can be represented in terms of the functions $\varphi$ and $P_{m}$ introduced in (1.7) and (1.8), respectively. To be explicit, we have

$$
K_{m}(z)=\frac{1}{2}+P_{m}(\varphi(z))
$$

and also

$$
K_{m}(z)=-\frac{1}{2}-P_{m}(\varphi(-z)) .
$$

Proof. In fact, for $z \neq n \pi \quad(n=0, \pm 1, \ldots)$,

$$
K_{m}(z)=\frac{(2 m+1) !}{2^{2 m+1}(m !)^{2}} \int_{0}^{-i \cot \pi z}\left(1-t^{2}\right)^{m} d t,
$$

as is seen by applying the binomial theorem under the integral sign and integrating term by term. Noting that $-i \cot \pi z=1-2 \varphi(z)$, we obtain

$$
\int_{0}^{-i \cot \pi z}\left(1-t^{2}\right)^{m} d t=\int_{0}^{1}\left(1-t^{2}\right)^{m} d t+\int_{1}^{1-2 \varphi(z)}\left(1-t^{2}\right)^{m} d t .
$$

The first integral on the right-hand side is equal to $2^{2 m}(m !)^{2} /(2 m+1)$ !, whereas the second one may be written as

$$
\int_{1}^{1-2 \varphi(=)}\left(1-t^{2}\right)^{m} d t=-2^{2 m+1} \int_{0}^{\varphi(z)} u^{m}(1-u)^{m} d u .
$$

Hence (3.8) holds. The representation (3.9) follows since $K_{m}$ is an odd function of $z$.

The relevance of the function $K_{m}$ is clearly demonstrated by the next lemma. 
Lemma 2. Let $n$ be any integer and let $f$ be holomorphic in a neighborhood of $n$. Then

$$
\sum_{\mu=0}^{m} c_{m, 2 \mu} f^{(2 \mu)}(n)=2 \pi i \operatorname{Res}\left(K_{m}(z) \cdot f(z)\right)_{z=n} .
$$

Proof. In view of the periodicity of $K_{m}$ it is enough to prove the statement for $n=0$. Since $K_{m}$ has a Laurent series development of the form

$$
K_{m}(z)=\sum_{\mu=-m}^{\infty} b_{m, 2 \mu-1} z^{2 \mu-1} \quad(0<|z|<1),
$$

we readily obtain

$$
\operatorname{Res}\left(K_{m}(z) \cdot f(z)\right)_{z=0}=\sum_{\mu=0}^{m} \frac{1}{(2 \mu) !} b_{m,-2 \mu-1} f^{(2 \mu)}(0) .
$$

Now if we set

$$
\widetilde{Q}_{m}(x):=2 \pi i \sum_{\mu=0}^{m} \frac{1}{(2 \mu) !} b_{m,-2 \mu-1} x^{2 \mu},
$$

then it will be enough to show that $\widetilde{Q}_{m}(x) \equiv Q_{m}(x)$.

Applying (3.11) to $f(z)=e^{i 2 \pi j z}$, we obtain

$$
\operatorname{Res}\left(K_{m}(z) \cdot e^{i 2 \pi j z}\right)_{z=0}=\widetilde{Q}_{m}(i 2 \pi j) .
$$

On the other hand,

$$
\operatorname{Res}\left(K_{m}(z) \cdot e^{i 2 \pi j z}\right)_{z=0}=\frac{1}{2 \pi i} \int_{\Gamma} K_{m}(z) e^{i 2 \pi j z} d z,
$$

where $\Gamma$ is the positively oriented boundary of the rectangle with vertices at $\pm \frac{1}{2} \pm i T(T>0)$. Since the integrand in (3.13) has period 1 , the contributions coming from the two vertical sides of $\Gamma$ cancel. Furthermore, using the representation (3.8) for $K_{m}(z)$ in the lower half-plane, and (3.9) in the upper half-plane, we find that the contributions coming from the horizontal parts of $\Gamma$ tend to zero as $T \rightarrow+\infty$ provided $j \in\{ \pm 1, \ldots, \pm m\}$. Hence, (3.12) and (3.13) yield that

$$
\widetilde{Q}_{m}(i 2 \pi j)=0 \text { for } j= \pm 1, \ldots, \pm m
$$

and consequently

$$
\frac{(2 m) !}{2 \pi i b_{m,-2 m-1}} \widetilde{Q}_{m}(x) \equiv(2 \pi)^{2 m}(m !)^{2} Q_{m}(x) .
$$

From the definition of $K_{m}$ we find that

$$
b_{m,-2 m-1}=-i \frac{(2 m) !}{(2 \pi)^{2 m+1}(m !)^{2}},
$$

and so (3.14) reduces to $\widetilde{Q}_{m}(x) \equiv Q_{m}(x)$, which completes the proof of Lemma 2.

As stated, Theorem $\mathrm{C}$ deals with $E_{0}[M, N ; f]$ only, but it is possible to deduce from (2.2) a representation for $E_{m}[M, N ; f]$. In fact, there is a very simple relationship between $E_{m}[M, N ; f]$ and $E_{0}[M, N ; f]$ and also 
between $E_{m}\left[M-\frac{1}{2}, N-\frac{1}{2} ; f\right]$ and $E_{0}\left[M-\frac{1}{2}, N-\frac{1}{2} ; f\right]$. To be precise, we have

Lemma 3. Under the assumptions of Theorems 1 and 2 , respectively, and in the notations of (1.1), (1.4), (1.5) and (1.6) we have

$$
\begin{aligned}
& E_{m}\left[M-\frac{1}{2}, N-\frac{1}{2} ; f\right]= E_{0}\left[M-\frac{1}{2}, N-\frac{1}{2} ; f_{m}\right] \\
&-\sum_{\mu=1}^{m} c_{m, 2 \mu}\left\{f^{(2 \mu-1)}\left(N-\frac{1}{2}\right)-f^{(2 \mu-1)}\left(M-\frac{1}{2}\right)\right\}, \\
& E_{m}[M, N ; f]=E_{0}\left[M, N ; f_{m}\right]-\sum_{\mu=1}^{m} c_{m, 2 \mu}\left\{f^{(2 \mu-1)}(N)-f^{(2 \mu-1)}(M)\right\} .
\end{aligned}
$$

Proof. We have

$$
\begin{aligned}
E_{0}[M & \left.-\frac{1}{2}, N-\frac{1}{2} ; f_{m}\right]=\int_{M-1 / 2}^{N-1 / 2} \sum_{\mu=0}^{m} c_{m, 2 \mu} f^{(2 \mu)}(x) d x-\sum_{n=M}^{N-1} f_{m}(n) \\
& =\left\{\int_{M-1 / 2}^{N-1 / 2} f(x) d x-\sum_{n=M}^{N-1} f_{m}(n)\right\}+\sum_{\mu=1}^{m} c_{m, 2 \mu} \int_{M-1 / 2}^{N-1 / 2} f^{(2 \mu)}(x) d x \\
& =E_{m}\left[M-\frac{1}{2}, N-\frac{1}{2} ; f\right]+\sum_{\mu=1}^{m} c_{m, 2 \mu}\left\{f^{(2 \mu-1)}\left(N-\frac{1}{2}\right)-f^{(2 \mu-1)}\left(M-\frac{1}{2}\right)\right\},
\end{aligned}
$$

which proves the first of the two identities. The second is proved in the same way.

The next three lemmas will be useful in our study of the integrals appearing on the right-hand sides of (2.1) and (2.2).

Lemma 4. Let $f$ be holomorphic and of exponential type less than $2 \pi(m+1)$ in the right half-plane such that $f(x) \rightarrow 0$ as $x \rightarrow+\infty$. Then with $P_{m}$ as in (1.8),

$$
\lim _{N \rightarrow+\infty} \int_{0}^{+\infty} P_{m}\left(\frac{1}{1+e^{2 \pi t}}\right)\{f(N+\lambda+i t)-f(N+\lambda-i t)\} d t=0
$$

uniformly for $\lambda \in\left[\frac{1}{2}, \frac{3}{2}\right]$.

Proof. Since $f(x) \rightarrow 0$ as $x \rightarrow+\infty$, the function $f$ is bounded on the positive real axis and so [1, Theorem 6.2.3]

$$
|f(x+i t)| \leq c e^{\sigma|t|}, \quad 0<x<+\infty, t \in \mathbf{R},
$$

for some $c>0$ and some $\sigma<2 \pi(m+1)$. Thus, taking (1.8) into account, there exists for given $\varepsilon>0$ an $\omega>0$ such that

$$
\left|\int_{\omega}^{+\infty} P_{m}\left(\frac{1}{1+e^{2 \pi t}}\right)\{f(N+\lambda+i t)-f(N+\lambda-i t)\} d t\right|<\frac{\varepsilon}{2}
$$


whenever $N+\lambda>0$. Furthermore, by a theorem of Montel [1, Theorem 1.4.9], $f(x+i t)$ tends to zero as $x \rightarrow+\infty$ uniformly for $t \in[-\omega, \omega]$. Hence there exists an $N_{0}>0$ such that

$$
\left|\int_{0}^{\omega} P_{m}\left(\frac{1}{1+e^{2 \pi t}}\right)\{f(N+\lambda+i t)-f(N+\lambda-i t)\} d t\right|<\frac{\varepsilon}{2}
$$

for all $N>N_{0}$ and $\lambda \in\left[\frac{1}{2}, \frac{3}{2}\right]$. With this, the proof of our assertion is complete.

Lemma 5. Let $L$ be the straight line segment joining the points $\pm i T$ and let $g: L \rightarrow \mathbf{C}$ be $k$ times continuously differentiable. Then

$$
\begin{aligned}
i \int_{0}^{T} P_{0} & \left(\frac{1}{1+e^{2 \pi t}}\right)\{g(i t)-g(-i t)\} d t \\
= & i \sum_{j=1}^{k} \Phi_{j}(-i T)\left\{g^{(j-1)}(i T)+(-1)^{j} g^{(j-1)}(-i T)\right\} \\
& +\sum_{j=1}^{[k / 2]} \frac{B_{2 j}}{(2 j) !}\left(1-2^{-2 j+1}\right) g^{(2 j-1)}(0) \\
& +\int_{0}^{T} \Phi_{k}(-i t)\left\{g^{(k)}(i t)-(-1)^{k} g^{(k)}(-i t)\right\} d t
\end{aligned}
$$

and

$$
\begin{aligned}
i \int_{0}^{T} P_{0} & \left(\frac{1}{1-e^{2 \pi t}}\right)\{g(i t)-g(-i t)\} d t \\
= & i \sum_{j=1}^{k} \Psi_{j}(-i T)\left\{g^{(j-1)}(i T)+(-1)^{j} g^{(j-1)}(-i T)\right\} \\
& -\sum_{j=1}^{[k / 2]} \frac{B_{2 j}}{(2 j) !} g^{(2 j-1)}(0) \\
& +\int_{0}^{T} \Psi_{k}(-i t)\left\{g^{(k)}(i t)-(-1)^{k} g^{(k)}(-i t)\right\} d t
\end{aligned}
$$

Proof. Noting that for $t>0$

$$
i P_{0}\left(\frac{1}{1+e^{2 \pi t}}\right)=\Phi_{0}(-i t)
$$

and

$$
i P_{0}\left(\frac{1}{1-e^{2 \pi t}}\right)=\Psi_{0}(-i t)
$$

the results follow by repeated integration by parts, using (3.4)-(3.7). 
Lemma 6. Let $g:[A, B] \rightarrow \mathbf{C}$ be $k$ times continuously differentiable; then in the notations of (1.7), (1.8) and (3.3) we have

$$
\begin{aligned}
\int_{A}^{B} & P_{0}(\varphi( \pm u-i T)) g(u) d u \\
= & i \sum_{j=1}^{k}(\mp 1)^{j}\left\{\Psi_{j}( \pm B-i T) g^{(j-1)}(B)-\Psi_{j}( \pm A-i T) g^{(j-1)}(A)\right\} \\
& -i( \pm 1)^{k} \int_{A}^{B} \Psi_{k}( \pm u-i T) g^{(k)}(u) d u
\end{aligned}
$$

Proof. Since

$$
P_{0}(\varphi(u))=-i \Psi_{0}(u),
$$

the result follows by repeated integration by parts, using the second formula of (3.4).

We also need the following lemma about functions holomorphic and of exponential type in a strip.

Lemma 7. If $f$ is holomorphic and of exponential type less than $\tau$ in the strip $S[a, b]$ defined in (2.14), then all the derivatives of $f$ are of exponential type less than $\tau$ in $S[a+\delta, b-\delta]$ for every $\delta \in(0,(b-a) / 2)$.

Proof. The result follows from the formula

$$
f^{(k)}(z)=\frac{k !}{2 \pi i} \int_{\gamma} \frac{f(\zeta)}{(\zeta-z)^{k+1}} d \zeta,
$$

taking as $\gamma$ the positively oriented circle with center at $z$ and radius $\delta$.

\section{Proofs of the Results STATEd IN $\S 2$}

Proof of Theorem 1. Let $\Gamma$ be the positively oriented boundary of $\mathscr{R}_{1}$. Then by the residue theorem and Lemma 2 we have

$$
\sum_{n=M}^{N-1} f_{m}(n)=\int_{\Gamma} K_{m}(z) f(z) d z .
$$

If $\Gamma_{1}$ denotes the part of $\Gamma$ lying in the closed upper half-plane and $\Gamma_{2}$ the rest, then using (3.8) for $z \in \Gamma_{2}$ and (3.9) for $z \in \Gamma_{1}$ we obtain

$$
\begin{aligned}
\int_{\Gamma} K_{m}(z) f(z) d z= & -\frac{1}{2} \int_{\Gamma_{1}} f(z) d z-\int_{\Gamma_{1}} P_{m}(\varphi(-z)) \cdot f(z) d z \\
& +\frac{1}{2} \int_{\Gamma_{2}} f(z) d z+\int_{\Gamma_{2}} P_{m}(\varphi(z)) \cdot f(z) d z
\end{aligned}
$$

But by Cauchy's theorem,

$$
-\int_{\Gamma_{1}} f(z) d z=\int_{M-1 / 2}^{N-1 / 2} f(x) d x=\int_{\Gamma_{2}} f(z) d z
$$


and so

$$
\begin{aligned}
\int_{\Gamma} K_{m}(z) f(z) d z= & \int_{M-1 / 2}^{N-1 / 2} f(x) d x-\int_{\Gamma_{1}} P_{m}(\varphi(-z)) f(z) d z \\
& +\int_{\Gamma_{2}} P_{m}(\varphi(z)) f(z) d z .
\end{aligned}
$$

Now, on splitting $\Gamma_{1}, \Gamma_{2}$ into their horizontal and vertical parts and combining the terms appropriately, we easily arrive via (4.1) at formula (2.1).

Proof of Theorem 2. It is enough to prove (2.3) for $a=-\frac{1}{2}, b=N-\frac{1}{2}, h=1$ and apply the result to $f\left(a+h\left(x+\frac{1}{2}\right)\right)$. Furthermore, given $\varepsilon>0$, using the approximation theorem of Weierstrass, we find a polynomial $p$ such that

$$
\max _{-1 / 2 \leq x \leq N-1 / 2}\left|f^{(j)}(x)-p^{(j)}(x)\right| \leq \varepsilon
$$

for $j=0, \ldots, 2 k$. Hence we may assume $f$ to be holomorphic in

$$
\left\{z \in \mathbf{C}:-\frac{1}{2} \leq \operatorname{Re} z \leq N-\frac{1}{2},|\operatorname{Im} z| \leq T\right\}, \quad T>0 .
$$

Applying Theorem 1 with $M=0$ and $m=0$, we obtain for $T \rightarrow 0$

$$
\int_{-1 / 2}^{N-1 / 2} f(x) d x-\sum_{n=0}^{N-1} f(n)=-\int_{-1 / 2}^{N-1 / 2}\left\{P_{0}(\varphi(u))+P_{0}(\varphi(-u))\right\} f(u) d u .
$$

By Lemma 6 with $k$ replaced by $2 k$, the right-hand side is equal to

$$
\begin{aligned}
& -\left.i \sum_{j=1}^{2 k}\left\{(-1)^{j} \Psi_{j}(u)+\Psi_{j}(-u)\right\} f^{(j-1)}(u)\right|_{-1 / 2} ^{N-1 / 2} \\
& \quad+i \int_{-1 / 2}^{N-1 / 2}\left\{\Psi_{2 k}(u)+\Psi_{2 k}(-u)\right\} f^{(2 k)}(u) d u .
\end{aligned}
$$

With the help of (3.5) and (3.7) we get

$$
\begin{aligned}
E_{0}[ & \left.-\frac{1}{2}, N-\frac{1}{2} ; f\right] \\
= & \sum_{j=1}^{k} \frac{B_{2 j}}{(2 j) !}\left(1-2^{-2 j+1}\right)\left\{f^{(2 j-1)}\left(N-\frac{1}{2}\right)-f^{(2 j-1)}\left(-\frac{1}{2}\right)\right\} \\
& +\int_{-1 / 2}^{N-1 / 2} f^{(2 k)}(x) \frac{\widehat{B}_{2 k}(x)}{(2 k) !} d x .
\end{aligned}
$$

This can be used to obtain a representation for $E_{m}\left[-\frac{1}{2}, N-\frac{1}{2} ; f\right]$, since by Lemma 3

$$
\begin{aligned}
& E_{m}\left[-\frac{1}{2}, N-\frac{1}{2} ; f\right] \\
&=E_{0}\left[-\frac{1}{2}, N-\frac{1}{2} ; f\right]+\sum_{\mu=1}^{m} c_{m, 2 \mu}\left\{E_{0}\left[-\frac{1}{2}, N-\frac{1}{2} ; f^{(2 \mu)}\right]\right. \\
&\left.\quad-f^{(2 \mu-1)}\left(N-\frac{1}{2}\right)+f^{(2 \mu-1)}\left(-\frac{1}{2}\right)\right\} .
\end{aligned}
$$


Applying (4.2) to $f^{(2 \mu)}$ with $k$ replaced by $k-\mu$, we obtain

$$
\begin{aligned}
E_{0}[ & \left.-\frac{1}{2}, N-\frac{1}{2} ; f^{(2 \mu)}\right]-f^{(2 \mu-1)}\left(N-\frac{1}{2}\right)+f^{(2 \mu-1)}\left(-\frac{1}{2}\right) \\
= & \sum_{j=0}^{k-\mu} \frac{B_{2 j}}{(2 j) !}\left(1-2^{-2 j+1}\right)\left\{f^{(2 \mu+2 j-1)}\left(N-\frac{1}{2}\right)-f^{(2 \mu+2 j-1)}\left(-\frac{1}{2}\right)\right\} \\
& +\int_{-1 / 2}^{N-1 / 2} f^{(2 k)}(x) \frac{\widehat{B}_{2 k-2 \mu}(x)}{(2 k-2 \mu) !} d x \\
= & \sum_{j=\mu}^{k} \frac{B_{2 j-2 \mu}}{(2 j-2 \mu) !}\left(1-2^{-2 j+2 \mu+1}\right)\left\{f^{(2 j-1)}\left(N-\frac{1}{2}\right)-f^{(2 j-1)}\left(-\frac{1}{2}\right)\right\} \\
& +\int_{-1 / 2}^{N-1 / 2} f^{(2 k)}(x) \frac{\widehat{B}_{2 k-2 \mu}(x)}{(2 k-2 \mu) !} d x,
\end{aligned}
$$

and so

$$
\begin{aligned}
\sum_{\mu=1}^{m} c_{m, 2 \mu}\left\{E_{0}\left[-\frac{1}{2}, N-\frac{1}{2} ; f^{(2 \mu)}\right]-f^{(2 \mu-1)}\left(N-\frac{1}{2}\right)+f^{(2 \mu-1)}\left(-\frac{1}{2}\right)\right\} \\
=\sum_{j=1}^{k}\left\{f^{(2 j-1)}\left(N-\frac{1}{2}\right)-f^{(2 j-1)}\left(-\frac{1}{2}\right)\right\} \\
\quad \times \sum_{\mu=1}^{\min \{m, j\}} \frac{B_{2 j-2 \mu}}{(2 j-2 \mu) !}\left(1-2^{-2 j+2 \mu+1}\right) c_{m, 2 \mu} \\
\quad+\int_{-1 / 2}^{N-1 / 2} f^{(2 k)}(x) \sum_{\mu=1}^{m} c_{m, 2 \mu} \frac{\widehat{B}_{2 k-2 \mu}(x)}{(2 k-2 \mu) !} d x
\end{aligned}
$$

Adding each side of (4.4) to the corresponding side of (4.2) and taking note of (4.3), we obtain

$$
\begin{aligned}
E_{m}\left[-\frac{1}{2}, N-\frac{1}{2} ; f\right] \\
=\sum_{j=1}^{k}\left\{f^{(2 j-1)}\left(N-\frac{1}{2}\right)-f^{(2 j-1)}\left(-\frac{1}{2}\right)\right\} \\
\quad \times \sum_{\mu=0}^{\min \{m, j\}} \frac{B_{2 j-2 \mu}}{(2 j-2 \mu) !}\left(1-2^{-2 j+2 \mu+1}\right) c_{m, 2 \mu} \\
\quad+\int_{-1 / 2}^{N-1 / 2} f^{(2 k)}(x) \sum_{\mu=0}^{m} c_{m, 2 \mu} \frac{\widehat{B}_{2 k-2 \mu}(x)}{(2 k-2 \mu) !} d x,
\end{aligned}
$$

which is equivalent to $(2.3)$.

The proof of Theorem 3 is analogous to that of Theorem 2, and so we omit it. 
Proof of Corollary 1. Using (3.5) and (1.1), we find that

$$
M_{k, m}(x)=(-1)^{k+1} 2 \sum_{\nu=m+1}^{\infty} \frac{\cos 2 \pi \nu x}{(2 \pi \nu)^{2 k}} Q_{m}(i 2 \pi \nu) .
$$

Starting now with

$$
R_{2, k, m, N}[f]=h^{2 k} \int_{a}^{b} f^{(2 k)}(x) M_{k, m}\left(\frac{x-a}{h}\right) d x,
$$

two integrations by parts yield

$$
\begin{aligned}
& R_{2, k, m, N}[f] \\
& \quad=-h^{2 k+2} \int_{a}^{b}\left\{M_{k+1, m}(0)-M_{k+1, m}\left(\frac{x-a}{h}\right)\right\} f^{(2 k+2)}(x) d x .
\end{aligned}
$$

From (4.5) we readily see that

$$
\left|M_{k, m}(x)\right| \leq\left|M_{k, m}(0)\right|
$$

for all $x \in \mathbf{R}$ and $k \geq m$. Hence, the term in curled brackets on the right-hand side of (4.6) does not change sign. Therefore, applying the mean value theorem for integrals,

$$
\begin{aligned}
R_{2, k, m, N}[f] & =-h^{2 k+2} f^{(2 k+2)}(\xi) \int_{a}^{b}\left\{M_{k+1, m}(0)-M_{k+1, m}\left(\frac{x-a}{h}\right)\right\} d x \\
& =(a-b) h^{2 k+2} f^{(2 k+2)}(\xi) M_{k+1, m}(0) .
\end{aligned}
$$

This is (2.6).

Using (2.26), we obtain the estimate

$$
\left|M_{k+1, m}(0)\right| \leq \frac{2}{(m !)^{2} \cdot(2 \pi)^{2 k+2}} \sum_{\nu=m+1}^{\infty} \frac{1}{\nu^{2 k+2-2 m}} .
$$

Furthermore,

$$
\begin{aligned}
\sum_{\nu=m+1}^{\infty} \frac{1}{\nu^{2 k+2-2 m}} & \leq \frac{1}{(m+1)^{2 k+2-2 m}}+\int_{m+1}^{\infty} \frac{1}{x^{2 k+2-2 m}} d x \\
& =\frac{2 k+2-m}{2 k+1-2 m} \frac{1}{(m+1)^{2 k+2-2 m}} .
\end{aligned}
$$

This shows how (2.7) follows from (2.6).

Proof of Theorem 4. The existence of the limit in (2.8) implies [1, Theorem 11.3.4*] that $f(x) \rightarrow 0$ as $x \rightarrow+\infty$, and so (3.15) holds for some $c>0$ and some $\sigma<2 \pi(m+1)$. Further, from (1.7) and (1.8) it follows that $\left|P_{m}(\varphi(u-i T))\right|$ and $\left|P_{m}(\varphi(-u-i T))\right|$ behave like

$$
\frac{(2 m+1) !}{(m+1) ! m !} e^{-2 \pi(m+1) T} \text { as } T \rightarrow+\infty
$$


Hence, Theorem 1 applied to $f(\cdot+\lambda)$ gives

$$
\begin{aligned}
& \sum_{n=1}^{N-1} f_{m}(n+\lambda) \\
& =\int_{1 / 2}^{N-1 / 2} f(x+\lambda) d x-i \int_{0}^{+\infty} P_{m}\left(\frac{1}{1+e^{2 \pi t}}\right) \\
& \quad \times\left\{f\left(N-\frac{1}{2}+\lambda+i t\right)-f\left(N-\frac{1}{2}+\lambda-i t\right)\right. \\
& \left.\quad-f\left(\frac{1}{2}+\lambda+i t\right)+f\left(\frac{1}{2}+\lambda-i t\right)\right\} d t .
\end{aligned}
$$

Now the desired result follows from Lemma 4.

Proof of Theorem 5. Starting with (4.7) for $\lambda=0$ and using Lemma 4, we can conclude this time that

$$
\lim _{N \rightarrow+\infty} \int_{1 / 2}^{N-1 / 2} f(x) d x
$$

exists for $N \in \mathbf{N}$. This and the fact that $f(x) \rightarrow 0$ as $x \rightarrow+\infty$ imply the existence of (2.8).

Proof of Corollary 2. Let $g(z):=f^{\prime}(z+1)$. Then by Lemma 7, $g$ is holomorphic and of exponential type less than $2 \pi$ in the right half-plane. If $f(x)$ tends to a limit as $x \rightarrow+\infty$, then

$$
\int_{1 / 2}^{+\infty} g(x) d x
$$

exists in the sense of Cauchy. Hence, applying Theorem 4 with $m=0$ and $f$ replaced by $g$, we obtain the uniform convergence of (1.3). Conversely, if (1.3) converges uniformly, then $g(x) \rightarrow 0$ as $x \rightarrow+\infty$, and the desired result follows from Theorem 5 .

Proof of Corollary 4. The first statement follows by applying Corollary 3 to the function $f(z)+f(-z)$. For the proof of (2.13) we may use Theorem 1 directly with $M=-N+1$. Letting first $T \rightarrow+\infty$ and then $N \rightarrow+\infty$, the result follows by taking into account (1.8), Lemma 4 and the estimate (3.15) for $f(z)+f(-z)$.

Proof of Theorem 6. It is enough to prove the theorem for $a=-\frac{1}{2}, b=N-\frac{1}{2}$, $h=1$. Let us first consider the case $m=0$. Applying Theorem 1 with $M=0$ and expressing the integrals on the right-hand side of (2.1) with the help of Lemmas 5 and 6 and with $k$ replaced by $2 k$, we find

$$
\begin{aligned}
& E_{0}\left[-\frac{1}{2}, N-\frac{1}{2} ; f\right]=\int_{-1 / 2}^{N-1 / 2} f(x) d x-\sum_{n=0}^{N-1} f(n) \\
& =i \sum_{j=1}^{2 k} \Phi_{j}(-i T)\left\{f^{(j-1)}\left(N-\frac{1}{2}+i T\right)+(-1)^{j} f^{(j-1)}\left(N-\frac{1}{2}-i T\right)\right. \\
& \left.\quad-f^{(j-1)}\left(-\frac{1}{2}+i T\right)-(-1)^{j} f^{(j-1)}\left(-\frac{1}{2}-i T\right)\right\} \\
& +\sum_{j=1}^{k} \frac{B_{2 j}}{(2 j) !}\left(1-2^{-2 j+1}\right)\left\{f^{(2 j-1)}\left(N-\frac{1}{2}\right)-f^{(2 j-1)}\left(-\frac{1}{2}\right)\right\} \quad \text { (continues) }
\end{aligned}
$$


(continued)

$$
\begin{aligned}
& +\int_{0}^{T} \Phi_{2 k}(-i t)\left\{f^{(2 k)}\left(N-\frac{1}{2}+i t\right)-f^{(2 k)}\left(N-\frac{1}{2}-i t\right)\right. \\
& \left.-f^{(2 k)}\left(-\frac{1}{2}+i t\right)+f^{(2 k)}\left(-\frac{1}{2}-i t\right)\right\} d t \\
& -i \sum_{j=1}^{2 k}(-1)^{j}\left\{\Psi_{j}\left(N-\frac{1}{2}-i T\right) \cdot f^{(j-1)}\left(N-\frac{1}{2}-i T\right)\right. \\
& +i \int_{-1 / 2}^{N-1 / 2} \Psi_{2 k}(u-i T) f^{(2 k)}(u-i T) d u \\
& -i \sum_{j=1}^{2 k}\left\{\Psi_{j}\left(-N+\frac{1}{2}-i T\right) \cdot f^{(j-1)}\left(-\frac{1}{2}-i T\right\}\right. \\
& +i \int_{-1 / 2}^{N-1 / 2} \Psi_{2 k}(-u-i T) f^{(2 k-1)}\left(N-\frac{1}{2}+i T\right) \\
& +\sum_{j=1} \frac{B_{2 j}}{(2 j) !}\left(1-\Psi_{j}\left(\frac{1}{2}-i T\right) f^{(j-1)}\left(-\frac{1}{2}+i T\right)\right\} \\
& +i \int_{-1 / 2}^{N-1 / 2}\left\{\Psi_{2 k}(u-i T) f^{(2 k)}(u-i T)\right. \\
& +\int_{0}^{T} \Phi_{2 k}(-i t)\left\{f^{(2 k)}\left(N-\frac{1}{2}+i t\right)-f^{(2 k)}\left(N-\frac{1}{2}-i t\right)\right. \\
& +(2 k-1) \\
& \left.\left.\left.+N-\frac{1}{2}\right)-i t\right)+f^{(2 k)}\left(-\frac{1}{2}-i t\right)\right\} d t \\
& \left.+(2 j-1)\left(-\frac{1}{2}\right)\right\}
\end{aligned}
$$

since $\Phi_{j}(-i T)=\Psi_{j}\left(-N+\frac{1}{2}-i T\right)=\Psi_{j}\left(N-\frac{1}{2}-i T\right)=\Psi_{j}\left(\frac{1}{2}-i T\right)=\Psi_{j}\left(-\frac{1}{2}-i T\right)$. Applying this to $f^{(2 \mu)}$ with $k$ replaced by $k-\mu$, we obtain

$$
\begin{aligned}
E_{0}\left[-\frac{1}{2},\right. & \left.N-\frac{1}{2} ; f^{(2 \mu)}\right] \\
= & \sum_{j=1}^{k-\mu} \frac{B_{2 j}}{(2 j) !}\left(1-2^{-2 j+1}\right)\left\{f^{(2 \mu+2 j-1)}\left(N-\frac{1}{2}\right)-f^{(2 \mu+2 j-1)}\left(-\frac{1}{2}\right)\right\} \\
& +i \int_{-1 / 2}^{N-1 / 2}\left\{\Psi_{2 k-2 \mu}(u-i T) f^{(2 k)}(u-i T)\right. \\
& \left.\quad+\Psi_{2 k-2 \mu}(-u-i T) f^{(2 k)}(u+i T)\right\} d u \\
& +\int_{0}^{T} \Phi_{2 k-2 \mu}(-i t)\left\{f^{(2 k)}\left(N-\frac{1}{2}+i t\right)-f^{(2 k)}\left(N-\frac{1}{2}-i t\right)\right. \\
& \left.-f^{(2 k)}\left(-\frac{1}{2}+i t\right)+f^{(2 k)}\left(-\frac{1}{2}-i t\right)\right\} d t .
\end{aligned}
$$


Hence, by (4.3),

$$
\begin{aligned}
& E_{m}\left[-\frac{1}{2}, N-\frac{1}{2} ; f\right]=\int_{-1 / 2}^{N-1 / 2} f(x) d x-\sum_{n=0}^{N-1} f_{m}(n) \\
& =E_{0}\left[-\frac{1}{2}, N-\frac{1}{2} ; f\right] \\
& +\sum_{\mu=1}^{m} c_{m, 2 \mu}\left\{E_{0}\left[-\frac{1}{2}, N-\frac{1}{2} ; f^{(2 \mu)}\right]-f^{(2 \mu-1)}\left(N-\frac{1}{2}\right)+f^{(2 \mu-1)}\left(-\frac{1}{2}\right)\right\} \\
& =\sum_{\mu=0}^{m} c_{m, 2 \mu}\left[\sum_{j=1}^{k-\mu} \frac{B_{2 j}}{(2 j) !}\left(1-2^{-2 j+1}\right)\left\{f^{(2 \mu+2 j-1)}\left(N-\frac{1}{2}\right)-f^{(2 \mu+2 j-1)}\left(-\frac{1}{2}\right)\right\}\right. \\
& +i \int_{-1 / 2}^{N-1 / 2}\left\{\Psi_{2 k-2 \mu}(u-i T) f^{(2 k)}(u-i T)\right. \\
& \left.+\Psi_{2 k-2 \mu}(-u-i T) f^{(2 k)}(u+i T)\right\} d u \\
& +\int_{0}^{T} \Phi_{2 k-2 \mu}(-i t)\left\{f^{(2 k)}\left(N-\frac{1}{2}+i t\right)-f^{(2 k)}\left(N-\frac{1}{2}-i t\right)\right. \\
& \left.\left.-f^{(2 k)}\left(-\frac{1}{2}+i t\right)+f^{(2 k)}\left(-\frac{1}{2}-i t\right)\right\} d t\right] \\
& -\sum_{\mu=1}^{m} c_{m .2 \mu}\left\{f^{(2 \mu-1)}\left(N-\frac{1}{2}\right)-f^{(2 \mu-1)}\left(-\frac{1}{2}\right)\right\} \\
& =\sum_{j=1}^{k} \sum_{\mu=0}^{\min \{m, j\}} c_{m, 2 \mu} \frac{B_{2 j-2 \mu}}{(2 j-2 \mu) !}\left(1-2^{-2 j+2 \mu+1}\right) \\
& \times\left\{f^{(2 j-1)}\left(N-\frac{1}{2}\right)-f^{(2 j-1)}\left(-\frac{1}{2}\right)\right\} \\
& +i \int_{-1 / 2}^{N-1 / 2}\left[\left\{\sum_{\mu=0}^{m} c_{m, 2 \mu} \Psi_{2 k-2 \mu}(u-i T)\right\} f^{(2 k)}(u-i T)\right. \\
& \left.+\left\{\sum_{\mu=0}^{m} c_{m, 2 \mu} \Psi_{2 k-2 \mu}(-u-i T)\right\} f^{(2 k)}(u+i T)\right] d t \\
& +\int_{0}^{T}\left\{\sum_{\mu=0}^{m} c_{m, 2 \mu} \Phi_{2 k-2 \mu}(-i t)\right\}\left\{f^{(2 k)}\left(N-\frac{1}{2}+i t\right)-f^{(2 k)}\left(N-\frac{1}{2}-i t\right)\right. \\
& \left.-f^{(2 k)}\left(-\frac{1}{2}+i t\right)+f^{(2 k)}\left(-\frac{1}{2}-i t\right)\right\} d t \\
& =\sum_{j=1}^{k} A_{1,2 j}\left\{f^{(2 j-1)}\left(N-\frac{1}{2}\right)-f^{(2 j-1)}\left(-\frac{1}{2}\right)\right\}+C_{1}(T)+C_{2}(T),
\end{aligned}
$$


where

$$
\begin{aligned}
& C_{1}(T)=(-1)^{k+1} \sum_{\nu=m+1}^{\infty} \frac{e^{-2 \pi T \nu}}{(2 \pi \nu)^{2 k}} Q_{m}(i 2 \pi \nu) \int_{-1 / 2}^{N-1 / 2}\left\{e^{-2 \pi i \nu u} f^{(2 k)}(u-i T)\right. \\
& \left.-e^{2 \pi i \nu u} f^{(2 k)}(u+i T)\right\} d u
\end{aligned}
$$

and

$$
\begin{aligned}
C_{2}(T)=\int_{0}^{T} L_{1, k, m}(t)\left\{f ^ { ( 2 k ) } \left(N-\frac{1}{2}+\right.\right. & i t)-f^{(2 k)}\left(N-\frac{1}{2}-i t\right) \\
& \left.-f^{(2 k)}\left(-\frac{1}{2}+i t\right)+f^{(2 k)}\left(-\frac{1}{2}-i t\right)\right\} d t .
\end{aligned}
$$

In view of Lemma 7 , the function $f^{(2 k)}$ is of exponential type less than $2 \pi(m+1)$ in $S\left[-\frac{1}{2}, N-\frac{1}{2}\right]$. Hence, $C_{1}(T)$ tends to a limit as $T \rightarrow \infty$ and so does $C_{2}(T)$; in particular,

$$
\lim _{T \rightarrow \infty} C_{1}(T)=0 \text {. }
$$

Therefore, Theorem 6 holds.

The proof of Theorem 7 is completely analogous to that of Theorem 6, and so we omit it.

Proof of Corollary 8. Under our assumptions, $f^{(j)}(x) \rightarrow 0$ as $x \rightarrow \pm \infty$ (see [1, Theorem $\left.11.3 .4^{*}\right]$ ) for all $j \geq 0$. Now the result follows by applying Corollary 4 with $m=0$ to $f^{\prime}, f^{\prime \prime}$, etc. The series (2.28) not only exists as a Cauchy principal value but also converges in the usual sense, as is seen by using Corollary 3.

Proofs of Theorems 10 and 11 . The assumptions of Theorem 10 imply that

$$
|f(x+i y)|=o(x) \text { as } x \rightarrow+\infty
$$

uniformly for $|y|<y_{0}$, where $y_{0}$ is any positive number. In fact, if (2.30) holds, then setting

$$
F(x)=\int_{1 / 2}^{x} f(t) d t
$$

we conclude that

$$
|F(x)-\eta x|=o(x) \text { as } x \rightarrow+\infty
$$

for some constant $\eta$, and so Theorem $11.3 .4^{*}$ of [1] applied to

$$
(F(z)-\eta z) /(z+1)
$$

gives

$$
|f(x)|=o(x) \text { as } x \rightarrow+\infty .
$$

Now we obtain (4.8) by using Theorems 6.2.3 and 1.4.9 of [1]. The estimate (4.8) holds also in the situation of Theorem 11 , since (4.9) is one of the assumptions.

Applying Theorem 6.2 .3 of [1] to $f(z) /(1+z)$, we conclude from (4.8) that

$$
|f(x+i t)| \leq c|1+x+i t| e^{\sigma|t|} \quad \text { for } 0<x<+\infty, t \in \mathbf{R},
$$


where $c$ is a positive constant and $\sigma<2 \pi(m+1)$. Using (4.8) and (4.10), and arguing as in the proof of Lemma 4 , we find that

$$
\lim _{N \rightarrow+\infty} \frac{1}{N} \int_{0}^{+\infty} P_{m}\left(\frac{1}{1+e^{2 \pi t}}\right)\{f(N+\lambda+i t)-f(N+\lambda-i t)\} d t=0
$$

uniformly for $\lambda \in\left[\frac{1}{2}, \frac{3}{2}\right]$. With this, the proofs of Theorems 10 and 11 can be completed analogously to those of Theorems 4 and 5 .

Proof of Theorem 12. Let $\varepsilon>0$ and $\delta>0$ be given. We can choose real numbers $\gamma_{1}, \gamma_{2}, \ldots$ such that [5]

$$
\chi(z):=\prod_{n=1}^{\infty} \frac{\sin \gamma_{n} z}{\gamma_{n} z} \quad(\chi(0)=1)
$$

represents an entire function of exponential type $\delta$ satisfying

$$
\chi(x)=O\left(\exp \left(-\frac{|x|}{(\log |x|)^{1+\varepsilon}}\right)\right) \text { as } x \rightarrow \pm \infty .
$$

By standard techniques (see [1, Chapter 6]) it can be shown that this law of decay holds in every strip parallel to the real line. Hence

$$
\Psi(z):=\chi(z+i) \cdot \overline{\chi(\bar{z}+i)}
$$

is an entire function of exponential type $2 \delta$ which is positive on the real line and

$$
\Psi(x)=O\left(\exp \left(-2 \frac{|x|}{(\log |x|)^{1+\varepsilon}}\right)\right) \text { as } x \rightarrow \pm \infty .
$$

It is therefore possible to find an entire function $\Psi$ which is positive on the real line and $\Psi \cdot f$ is of exponential type less than $\pi(k+1)$ such that $\Psi(x) \cdot f(x) \rightarrow 0$ as $x \rightarrow \pm \infty$. Now applying Corollary 4 to $d(\Psi(z) \cdot f(z)) / d z$ with $m=$ $(k-1) / 2$, we obtain

$$
0=\int_{-\infty}^{+\infty}(\Psi(x) \cdot f(x))^{\prime} d x=c_{m, 2 m} \sum_{n=-\infty}^{\infty} \Psi(n) \cdot f^{(k)}(n) .
$$

Since $c_{m, 2 m} \neq 0$ and $\Psi(n)>0$ for all $n$, we conclude that $f^{(k)}(n)=0$ for all $n \in \mathbf{Z}$. Hence $f(z) \equiv 0$ by Theorem $\mathrm{D}$.

Proof of Theorem 13. Under our assumptions all derivatives of $f(x)+f(-x)$ tend to zero as $x \rightarrow \pm \infty$. Thus, setting $m=(j-1) / 2$ and applying Corollary 4 to $f^{\prime \prime}$, we obtain

$$
0=\int_{-\infty}^{+\infty} f^{\prime \prime}(x) d x=c_{m, 2 m} \sum_{n=-\infty}^{\infty} f^{(j+1)}(n) .
$$

Since $c_{m .2 m} \neq 0$, it follows from $(2.32)$ that $f^{(j+1)}(n)=0$ for all $n \in \mathbf{Z}$. Arguing in a similar way with $f^{\left(2 \prime^{\prime}\right)}$, where $2 \nu \leq 2 k-j+1$, we successively find that the derivatives of $f$ of even order $j+1, \ldots, 2 k$ vanish for all $n \in \mathbf{Z}$. The conclusion then follows from Theorem $E$. 


\section{ACKNOWLEDGMENT}

We are grateful to Professor R. P. Boas for drawing our attention to his paper [2] with A. C. Schaeffer.

\section{BIBLIOGRAPHY}

1. R. P. Boas, Jr, Entire functions, Academic Press, New York, 1954.

2. R. P. Boas, Jr. and A. C. Schaeffer, New inequalities for entire functions, J. Math. Mech. 7 (1958), 191-201.

3. R. Brück, Identitätssätze für ganze Funktionen vom Exponentialtyp, Mitteilungen Math. Seminar Giessen, Heft 168, 1984.

4. J. D. Donaldson and D. Elliott, A unified approach to quadrature rules with asymptotic estimates of their remainders, SIAM J. Numer. Anal. 9 (1972), 573-602.

5. R. Gervais, Q. I. Rahman and G. Schmeisser, A bandlimited function simulating a durationlimited one, in Anniversary Volume on Approximation Theory and Functional Analysis (P. L. Butzer, R. L. Stens, B. Sz.-Nagy, eds.), ISNM vol. 65, Birkhäuser, Basel, 1984, pp. 355-362.

6. G. H. Hardy, Divergent series, Oxford Univ. Press, Oxford, 1949.

7. P. Henrici, Applied and computational complex analysis, vol. 1, Wiley, New York, 1974.

8. R. Kress, On the general Hermite cardinal interpolation, Math. Comp. 26 (1972), 925-933.

9. E. Lindelöf, Le calcul des résidus, Chelsea, New York, 1947.

10. J. McNamee, Error-bounds for the evaluation of integrals by the Euler-Maclaurin formula and by Gauss-type formulae, Math. Comp. 18 (1964), 368-381.

11. P. Olivier and Q. I. Rahman, Sur une formule de quadrature pour des fonctions entières, RAIRO Modél. Math. Anal. Numér. 20 (1986), 517-537.

12. G. Schmeisser and H. Schirmeier, Praktische Mathematik, de Gruyter, Berlin, 1976.

13. F. Stenger, Integration formulae based on the trapezoidal formula, J. Inst. Math. Appl. 12 (1973), 103-114.

14. _ Numerical methods based on Whittaker cardinal or sinc functions, SIAM Rev. 23 (1981), 165-224.

15. H. Takahasi and M. Mori, Estimation of errors in the numerical quadrature of analytic functions, Applicable Anal. 1 (1971), 201-229.

16. E. C. Titchmarsh, The theory of functions, 2nd ed., Oxford Univ. Press, Oxford, 1939.

Département de Mathématiques et de Statistique, Université de Montréal, Montréal, Québec, Canada H3C 3J7

Mathematisches Institut, Universität Erlangen-Nürnberg, Bismarckstrasse $1 \frac{1}{2}$, D-8520 ErLangen, West Germany 